\title{
Muestra académica de dibujo monumental
}

\author{
Lic. Venecia López \\ Docente de la Licenciatura en Artes Plásticas \\ a cargo de la materia de Dibujo Monumental
}

Dibujar es construir por medio de la línea un andamiaje para sostener la realidad. Es también proyectar, planear, traducir el mundo exterior, llevarlo al signo visual para aprehenderlo. Dibujar es comunicar mediante el lenguaje más antiguo. Dibujar es trazar una línea: el signo primigenio cargado de historia y de gestualidad.

En las paredes la Galería Sociedad Artesanos Hidalgo se exhibió una muestra de lo que se produce en las aulas de la clase de dibujo de figura humana monumental. La exposición se llevó a cabo del día 6 al 22 de Mayo del presente año. En ella se presentaron los trabajos finales de los alumnos Guillermo Carrillo, Martín Cotaque, Ray Erickson, Michelle Escarrega, Francisca Galaz, David Hernández, Dalila Mar, Luis Meleses, Arturo Ordaz, Altaire Navarro, Javier Olea, Carolina Perez, Daniela Plascencia, Rosa Román, Lizbeth Salazar, María Tarragona y Cristian Villegas. Los trabajos expuestos consistieron en proyectos de carácter monumental para espacios urbanos de la ciudad de Hermosillo.

El taller de dibujo monumental se convirtió en una suerte de laboratorio de producción desde el cual se reflexionó acerca del espacio y del entorno. se discutieron los imaginarios urbanos y los alumnos expusieron su ideas sobre la ciudad y su identidad. El propósito fue desarrollar el concepto de su propuesta por medio de investigación y de reflexión.
Para resolver la parte técnica se buscó proyectar en el papel craft, soporte sobre el cual se realizan las prácticas de taller, un dibujo en formato monumental que abordara la composición sugerida por los temas sobre los que reflexionaron.

La composición se nutrió por los diversos estudios de figura humana realizados en el taller, en los que el cuerpo es el objeto de estudio. Se profundiza en la anatomía, se aprende el movimiento, las posibilidades expresivas de la línea, los diferentes sistemas de representación, la figura en la historia del arte, el escorzo y la composición para al final realizar un dibujo de figura humana a gran escala.

Para comprender mejor el proceso de producción de piezas monumentales los alumnos estudiaron algunos ejemplos tomados de la historia del arte. Investigaron sobre artistas de diversas épocas y las es-

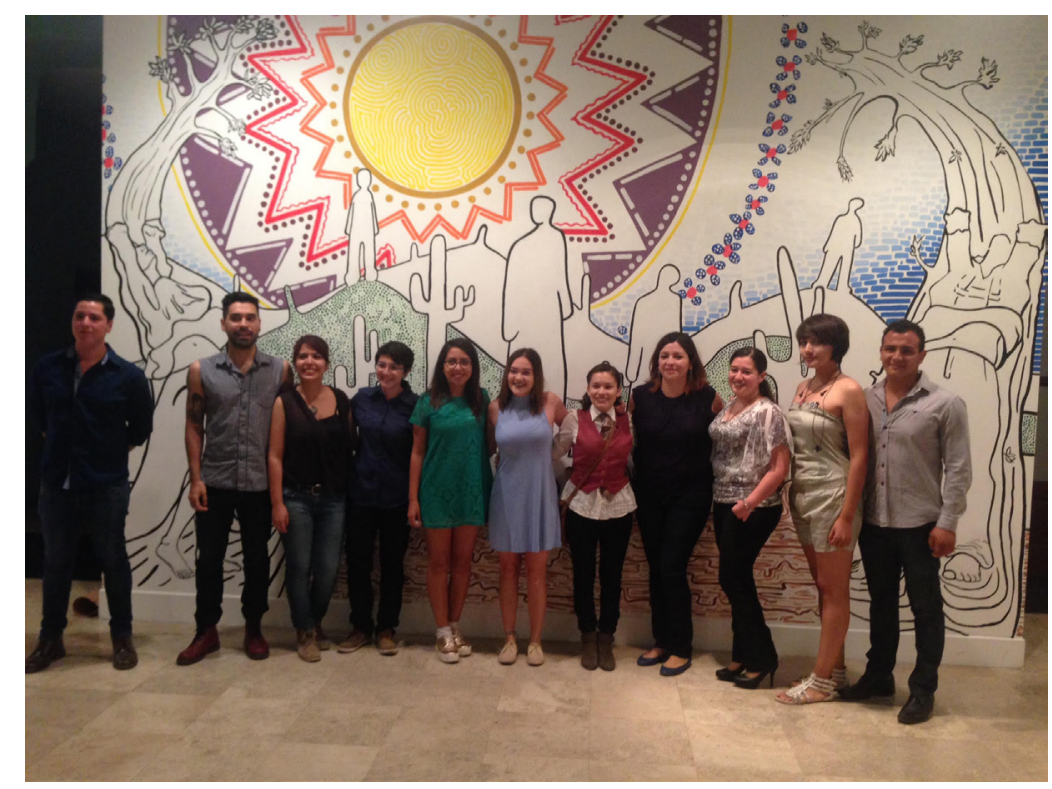


trategias que estos mismos utilizaron para la representación en gran formato; Miguel Ángel, por ejemplo cambiaba la proporción de los miembros de figura humana pensando en el punto de vista del espectador; Siqueiros empleó la poliangularidad para aportar dinamismo a la obra.

Para producir esta obra los alumnos salieron del taller a la calle en busca del muro ideal para proyectar en él las líneas que sustentarían sus temas. Una contundente serie de dibujos fue el resultado. También hicieron el montaje virtual en fotografía, y este se expuso a un lado de los ejercicios gráficos para poder evidenciar como cambiaría ese muro con el dibujo sobrepuesto.

Dentro de la galería existió por un momento otra propuesta de ciudad en la que sus espacios fueron tocados por la elocuencia del dibujo, y por toda la historia y la emoción de la línea que construye. La experiencia que ha dejado este proyecto se puede traducir en un primer acercamiento a la realización de un proyecto ejecutivo, también ha motivado al estudiante a investigar para sustentar una obra, y por supuesto, a bocetar en gran formato. Enhorabuena por esas grandes visiones que nutrirán el arte público en nuestra ciudad.

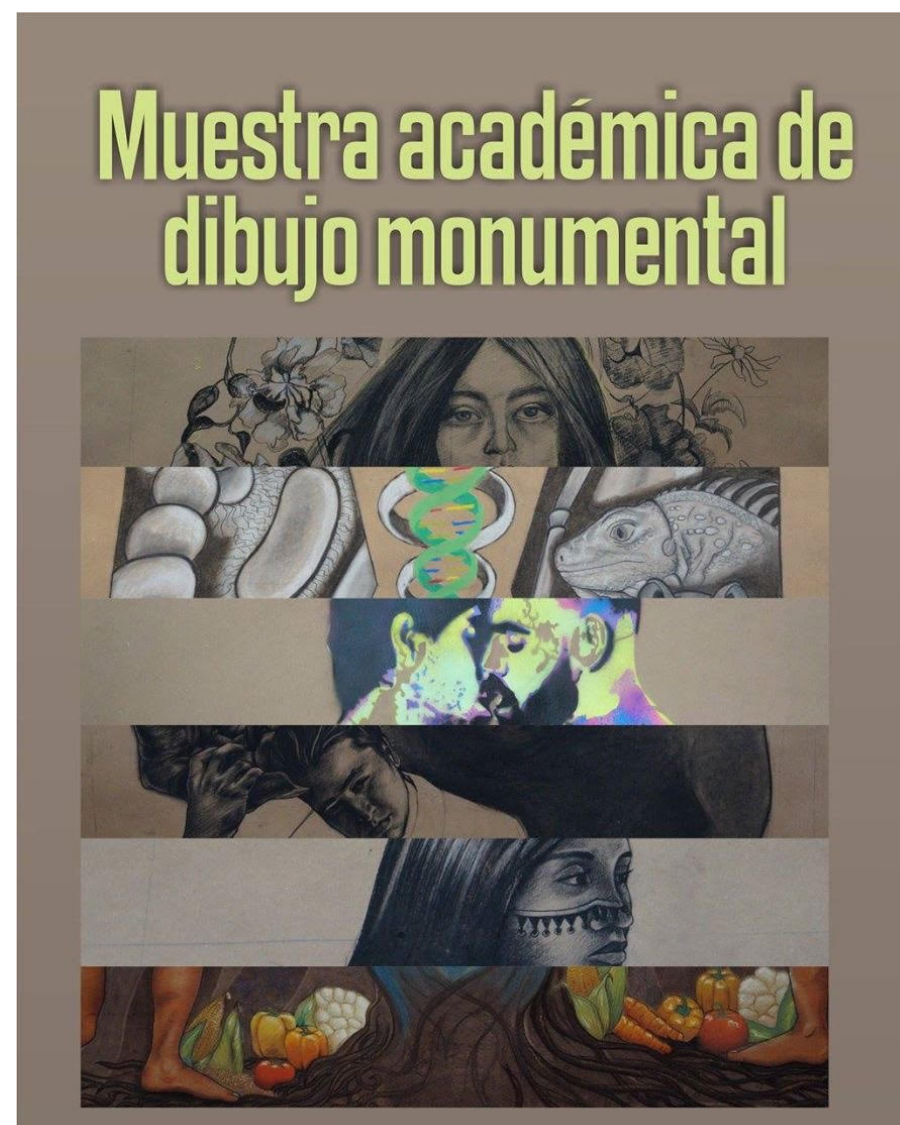

Inauguración: viernes 6 de Mayo de 2016 19:00 horas

Galería Sociedad de Artesanos Hidalgo (Monterrey y Garmendia, Col. Centro)

Estará expuesta hasta el 22 de Mayo Hermosillo, Sonora, 2016.

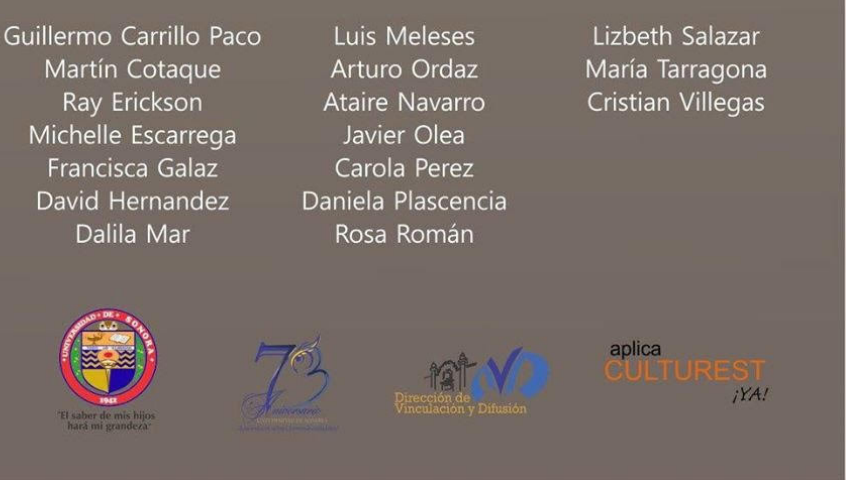

